\title{
SUPLEMENTOS DIETARIOS: ¿CUÁNTO SABEMOS PARA PODER USARLOS CORRECTAMENTE?
}

Cinthia C. Lutz ${ }^{1}$, Ma. Florencia Fernández ${ }^{1}$, César A. Lezcano ${ }^{1}$, Adriana L. Moresi ${ }^{1}$, Roxana I. Goyechea ${ }^{1}$, Diana C. Fechner ${ }^{1}$, Eva G.S. Miño ${ }^{2}$, Mgter. Mónica Esquivel2, Juan D. Ruíz Díaz ${ }^{1}$, Roberto G. Pellerano ${ }^{1 *}$

\section{RESUMEN}

Este proyecto aborda la problemática actual sobre el consumo indiscriminado de suplementos dietarios, que en general son percibidos como productos seguros y que sin embargo frente a un uso inadecuado pueden causar serias contraindicaciones. Las actividades planteadas buscan promover la reflexión, investigación y búsqueda de información relevante con respecto a los efectos beneficiosos y riesgos asumidos al consumir estos alimentos, a nivel de las comunidades involucradas (población objetivo): los alumnos de las instituciones educativas de nivel medio de la Ciudad de $\mathrm{Co}^{-}$ rrientes en las que se desarrollará el proyecto. La metodología propuesta para alcanzar los objetivos propuestos, propone la realización de actividades que permitan la interacción entre los alumnos de la universidad y los distintos integrantes de la comunidad educativa, principalmente los alumnos del nivel medio hacia quienes está destinado el proyecto de manera directa, mediante la reali- zación de seminarios de difusión y actualización de los conocimientos disponibles a realizarse en el colegio junto a los alumnos.

\section{PALABRAS CLAVE}

suplementos, vitaminas; minerales; proteínas; creatina

\section{INTRODUCCIÓN}

Los suplementos dietarios tienen por objetivo principal garantizar un consumo adecuado de nutrientes esenciales en la población en general; algunos suplementos pueden ayudar también a reducir el riesgo de contraer algunas enfermedades. No obstante, los suplementos no deberían reemplazar la variedad de alimentos que son importantes en una dieta saludable. A diferencia de los medicamentos, el objetivo de los suplementos no es el de tratar, diagnosticar, ni curar enfermedades. Eso significa que los suplementos no deben hacer afirmaciones tales como "reduce el dolor de la

\footnotetext{
(1) Dpto. de química, Facultad de Ciencias Exactas y Naturales y Agrimensura, UNNE, Av. Libertad 5.470, Corrientes, Argentina

(2) Colegio secundario “Dr. Luis Federico Leloir”, Avenida La Paz y Santa Cruz Cacique Canindeyu, Ctes, Argentina

*E-mail: roberto.pellerano@comunidad.unne.edu.ar (Director)
} 
artritis", o "sirve para el tratamiento de las enfermedades cardíacas". Estas afirmaciones sólo pueden hacerse de manera legítima para los medicamentos y no para los suplementos. En este contexto, los suplementos, utilizados en base a criterios científicos y técnicos, ofrecen importantes beneficios. Pero cuando se usan de forma inapropia$\mathrm{da}$, por incumplimiento o por automedicación pueden convertirse en riesgosos para la salud individual y colectiva, llegando a resultar ineficaces, producir efectos indeseables y hasta tóxicos. Los consumidores necesitan acceder a información veraz y comprensible acerca de los potenciales beneficios y riesgos de suplementos dietarios en general; cómo actúan en el organismo; las limitaciones de la farmacoterapia y otras opciones de tratamiento. El proyecto consiste en acercar información precisa y educar a la comunidad sobre distintos aspectos de los suplementos para mejorar la calidad de vida y hacer un uso racional de los mismos teniendo en cuenta que la utilización incorrecta de los suplementos disminuye sus beneficios aumentando los riesgos.

Desde hace más de una década, el perfil de los suplementos dietarios (tanto en la Argentina como en el resto del mundo) fue modificándose. Inicialmente eran sobre todo productos a base de vitaminas, minerales, proteínas, fibra, etc. En la actualidad, debido a los avances del conocimiento en el campo de la nutrición y en respuesta a las demandas de los consumidores, la oferta de productos a base de hierbas y otros nuevos nutrientes se encuentra en aumento. En la Argentina, los suplementos dietarios se encuentran incorporados al Código Alimentario Argentino (CAA) desde el año 1998. En el artículo 1381, son definidos como "productos destinados a incrementar la ingesta dietaria habitual, suplementando la incorporación de nutrientes en la dieta de las personas sanas que, no encontrándose en condiciones patológicas, presenten necesidades básicas dietarias no satisfechas o mayores a las habituales. Siendo su administración por vía oral, contenidas en envases que garanticen la calidad y estabilidad de los productos". En cuanto a su composición, deben aportar nutrientes, aunque también permite el uso de algunas hierbas, inicialmente sólo las incluidas en el CAA. Tal como se puede observar la legislación vigente se encuentra actualizada con respecto a las presentaciones disponibles en el mercado, sin embargo, resulta necesario crear conciencia con respecto al uso correcto de estos alimentos, dado que si son consumidos de manera errónea o imprudente pueden causar efectos biológicos perjudiciales.

En este contexto, los objetivos generales de este proyecto se proponen: (1) promover acciones de prevención de la salud, socializando conocimientos relacionados de gran vigencia actual, de manera de generar conciencia fundamentalmente en el uso racional de suplementos dietarios evitando la automedicación y el mal uso de los mismos; y (2) promover el desarrollo de la educación para la salud en los ámbitos de población juvenil del nivel medio, considerando este escenario, escuela, muy adecuado para un exitoso desarrollo de actividades preventivas hacia este sector de la población.

\section{METODOLOGÍA}

Este proyecto se propone difundir y concientizar con respecto al uso correcto de los suplementos dietarios disponibles en el mercado actualmente, y de esta forma brindar herramientas que permitan a los distintos integrantes de la comunidad tomar decisiones tendientes a mejorar la 
calidad de vida en general. La metodología propuesta para alcanzar los objetivos propuestos, propone la realización de actividades que permitan la interacción entre los alumnos de la universidad y los distintos integrantes de la comunidad educativa, principalmente los alumnos del nivel medio hacia quienes está destinado el proyecto de manera directa, mediante la realización de seminarios de difusión y actualización de los conocimientos disponibles a realizarse en el colegio junto a los alumnos.

Las actividades planteadas buscan estimular la reflexión, investigación y búsqueda de información relevante con respecto a los efectos beneficiosos y riesgos asumidos al consumir estos alimentos, a nivel de las comunidades involucradas: alumnos de las instituciones educativas de nivel medio de la Ciudad de Corrientes y comunidad educativa en las que se desarrollará el proyecto. En esta primera parte de ejecución del plan de trabajos se diseñaron y realizaron encuestas en tres franjas etarias de estudiantes del establecimiento educativo, con el objeto de generar información relevante que permita orientar acciones dirigidas a promover el uso racional de estos productos de acuerdo a las dudas e inquietudes manifestadas por los alumnos en estos instrumentos. Las encuestas fueron realizadas a un total de 40 individuos, entre 15 y 18 años, de los cuales un $67 \%$ eran varones. Las encuestas estaban compuestas por un total de 10 preguntas, fueron respondidas durante el horario de clases habitual de los alumnos y los resultados obtenidos se muestran a continuación. Es esperable que a partir de la información generada se puedan diseñar las presentaciones de difusión general, haciendo énfasis en las distintas informaciones de acuerdo a la franja etaria a la que se encuentren dirigidas.

\section{RESULTADOS Y DISCUSIÓN}

Las encuestas fueron realizadas a un total de 40 individuos, entre 15 y 18 años. Las figuras a continuación muestran gráficamente los resultados obtenidos para tres preguntas que consideramos más relevantes en el cuestionario.

\section{Riesgos al consumir suplementos}

= Ninguno $\quad$ Algunos $=$ Necesitan supervision médica



Figura 1. Respuestas obtenidas al preguntar sobre los tipos de riesgos que se asumen al consumir este tipo de alimentos. 


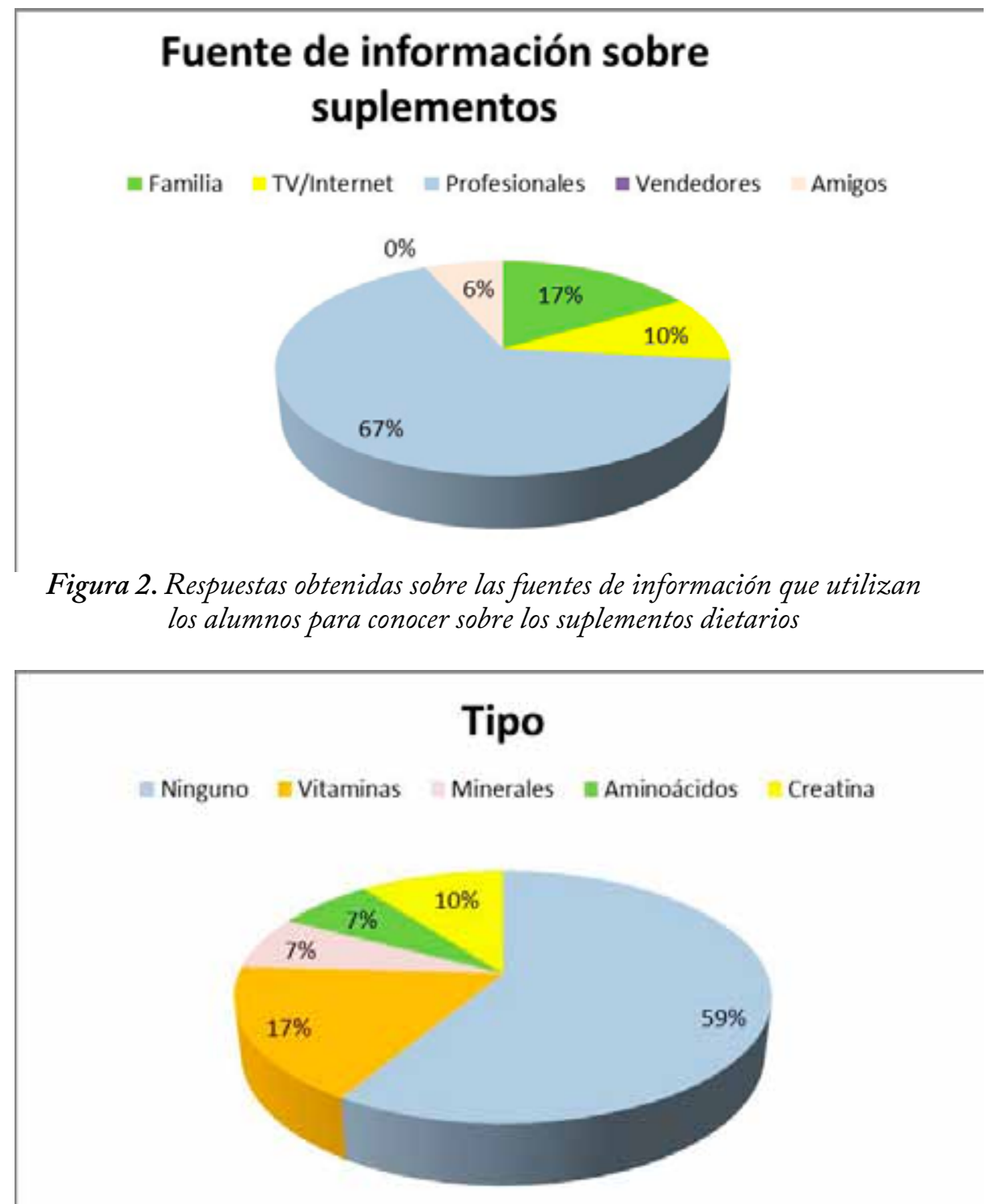

Figura 3. Respuestas obtenidas sobre qué tipos de suplementos dietarios son consumidos en caso de hacerlo.

Los resultados obtenidos indicaron que solo aproximadamente una tercera parte de los encuestados pudo definir correctamente lo que es un suplemento dietario (SD) y sus funciones (estos resultados no se muestran gráficamente), sin embargo, aproximadamente el $60 \%$ consideró que existen riesgos en el consumo de SDs (Fig. 1) y que el mismo debe ser supervisado por un profesio- nal de la salud (Fig. 2). Sobre esta última pregunta resulta importante aclarar, que no fueron incluidas entre las opciones a los instructores/entrenadores de gimnasios, y por esta razón algunos alumnos marcaron la opción de profesionales de la salud. A partir de esta sugerencia, decidimos modificar las opciones para esta pregunta para futuras encuestas. Adicionalmente, la gran mayoría 
de los encuestados consideró que los SDs pueden ser adquiridos en supermercados (no se muestran los resultados), y frente a la pregunta de si consume algún SDs, respondieron mayoritariamente que no, sin embargo, teniendo en cuenta el desconocimiento de la definición de SD, esta respuesta debe ser analizada en contexto. Finalmente, los alumnos que respondieron que consumían algún tipo de SD (Fig. 3), al ser preguntados sobre el tipo de $\mathrm{SD}$, aproximadamente un $17 \%$ de los encuestados reconocieron consumir SD a base de aminoácidos y/o creatina, generalmente con el objeto de mejorar el rendimiento deportivo. Estos resultados resultan preocupantes dado que la mayoría de los encuestados reconoció que consume estos productos sin realizarse chequeos o bajo supervisión médica.

\section{CONCLUSIONES}

Con estos resultados, los alumnos de la FaCENA, con la colaboración de los docentes del colegio secundario, se encuentran actualmente elaborando el material de difusión, en la búsqueda de generar conciencia fundamentalmente en el uso racional de SDs evitando la automedicación y el mal uso de los mismos. Los resultados aquí mostrados constituyen un informe de avance del desarrollo del proyecto de extensión "SUPLEMENTOS DIETARIOS: ¿CUÁNTO SABEMOS PARA PODER USARLOS CORRECTAMENTE?", proyecto financiado por el programa Universidad en el medio de la UNNE, convocatoria 2015.

\section{BIBLIOGRAFÍA}

- U.S. Food and Drug Administration (FDA). Dietarysupplements. http:// www.fda.gov/Food/DietarySupplements/ (Accedido 10/10/2016)
- ANMAT. Código Alimentario Argentino. Capítulo XVII. www.anmat. gov.ar/alimentos/codigoa/Capitulo_XVII.pdf (Accedido 10/10/2016) 